\title{
Laparoscopic Acute Care Surgery in Liver Disease: A Regression Analysis of the Nationwide Inpatient Sample
}

\author{
Sri Ram Pentakota ${ }^{1}$, Punam Patel $^{1}$ and Aziz M. Merchant ${ }^{1 *}$ \\ ${ }^{1}$ Department of Surgery, Rutgers-New Jersey Medical School, Newark, NJ 07103 USA
}

Received: 11 January, 2017; Accepted: 23 February, 2017; Published: 04 March, 2017

*Corresponding author: Aziz M. Merchant, Rutgers-New Jersey Medical School, 185 South Orange Avenue, Suite MSB G530, Newark, NJ 07103 USA, Tel no: 973 972-0072, Fax no: 973 972-6803; E-mail: Aziz.Merchant@rutgers.edu

\begin{abstract}
Introduction: Patients with liver disease are being more frequently undergoing urgent and emergent surgery. There is sparse literature comparing outcomes based on modality of surgery (laparoscopic versus open) in this important cohort of patients. Our study examines surgical outcomes by liver disease status and surgical modality in acute care surgery setting.

Methods: This is a retrospective cohort study of patients undergoing acute care abdominal surgery between 2006 and 2010 extracted from the National Inpatient Sample database. Surgical outcomes examined were in-hospital mortality, surgical, and medical complications, length of stay, and total hospital charges. Sequential multivariable generalized linear regression models were used to compare the outcomes across surgical modality and type of liver disease.

Results: Unadjusted and adjusted analyses revealed that laparoscopic surgery was associated with marked reduction in mortality (92\%) and surgical complications (86\%) compared to open surgery, as well as a moderate yet significant reduction $(60 \%)$ for medical complications, length of stay and total hospital charges. Patients with cirrhotic liver disease fared worse compared to those with non-cirrhotic and no liver disease, as expected.
\end{abstract}

Conclusion: Laparoscopic surgery provides a feasible alternative for patients with liver disease undergoing acute care surgery.

Keywords: Acute care surgery; Laparoscopic surgery; Surgical outcomes; Liver disease

\section{Introduction}

Patients with liver disease are increasingly undergoing acute care abdominal surgery, in part because of the long-term survival of patients with cirrhosis. The traditional approach for surgical treatment of this patient population has been through open surgery and laparotomy. There is limited evidence-based data on surgical outcomes vis-à-vis liver disease status in acute care abdominal surgery, and by extension even less data on outcomes stratified by surgical modality (laparoscopicvs open). Though liver resection for hepatic tumors and liver transplantation are the most common surgical procedures performed in cirrhotics, these patients frequently present with urgent and emergent non-hepatic abdominal surgical conditions such as cholecystitis, peptic ulcer disease and complicated hernias [1-4]. Overall, it has been estimated that $10 \%$ of patients with liver diseases undergo at least one operative procedure during the final 2 years of their lives $[5,6]$.

One of the most marked risks of poor outcomes in cirrhotic patients is the need for emergent surgical interventions [7]. Patients with compromised liver function are known to decompensate due to the stress of both anesthesia and surgery, and in spite of significant advances mortality and morbidity continue to remain high [8]. A recent series by Del Olmo, et al comparing 135 cirrhotic patients with 86 non cirrhotic patients, all undergoing non-hepatic general surgery, showed a $16.3 \%$ 1-month cirrhotic mortality compared with $3.5 \%$ for their control [9]. In addition, various complications affect the surgical outcome of these patients, including bleeding, infection, renal insufficiency, and multisystem organ failure [10]. The underlying liver dysfunction predisposes cirrhotic patients to coagulopathy resulting in excessive perioperative bleeding, hepatic hypoperfusion, septic complications, poor wound healing, and multiorgan dysfunction. In trauma settings, numerous retrospective studies have noted excessive mortality rates among cirrhotic patients compared with their non-cirrhotic counterparts [11-13].

Recently, laparoscopy has gained traction as an important adjunct in the surgical treatment of cirrhotic patients who require acute care surgery. Cirrhosis was considered an absolute contraindication to laparoscopy because of the risk for massive bleeding during dissection [14]. However, several case-series have recently reported success with laparoscopic acute care operations, e.g., laparoscopic cholecystectomy, in selected patients with cirrhosis [15-17]. One study reports 528 patients with cirrhosis who have undergone laparoscopic cholecystectomy. Most of these patients (81\%) are classified as Child-Pugh a cases, and only $1.1 \%$ are classified as C cases. The overall morbidity is $16 \%$, and the mortality rate is less than $1 \%$. These results compare favorably with those for open cholecystectomy and suggest that laparoscopic cholecystectomy can be performed 
safely for patients classified as Child-Pugh A and B cases. Though these reports, which happen to be predominantly case-series or single-center studies with a focus on one specific surgical procedure (i.e. cholecystectomy), demonstrate the safety and efficacy of laparoscopy in patients with cirrhotic liver disease, they have limited generalizability due to their scope and study design.

In our current study, we attempt to overcome some these limitations by using a large, national representative database, the National Inpatient Sample, to study surgical outcomes across all acute care abdominal surgeries and compare outcomes by patients' liver disease status and type of surgery (laparoscopic versus open). Furthermore, we compare outcomes by type of liver disease separately within each surgical modality, as well as analyze outcomes by surgical modality within each liver disease status cohort

\section{Materials and Methods}

\section{Data Source}

The National Inpatient Sample (NIS) databases were queried to answer our study questions. The NIS is the largest all-payer administrative database and is maintained as part of the Healthcare Cost and Utilization Project (HCUP) of the Agency for Healthcare Research and Quality (AHRQ) [18]. Through the HCUP program, the AHRQ brings together patient data collected by state-based organizations, hospital associations, and the federal government, as part of its focus on research of healthcare quality, costs, outcomes, and patient safety. The NIS discharge level data represents approximately 8 million hospital stays of an approximately $20 \%$ stratified sample of community, nonmilitary, and nonfederal hospitals in the United States. It contains information including admission day, admission source, patient and hospital characteristics, discharge destination and healthcare charges, and a number of important diagnosis and procedures. We used the NIS annual Core, Severity, and Hospital research files from 2006 to 2010 in this study.

\section{Study Population}

Our study population comprised of patients who underwent acute care abdominal surgery. We used the International Classification of Disease, Ninth Revision, Clinical Manifestation (ICD-9-CM) procedure codes, in position 1 to 15 , to first identify all patients that underwent any abdominal surgery which could be performed as urgent or emergency. The list of specific ICD9-CM procedure codes used is provided in Appendix1. Based on ICD-9-CM procedure codes, the type of surgery was coded either as laparoscopic or open surgery. Patients with codes indicating laparoscopic with conversion to open surgery were assigned to the open surgery group. From 2006 to 2010, we identified a total of $1,855,098$ patients based on the above ICD-9 procedure codes. We limited the study to adults by excluding patients who were < 18 years of age $(n=296,713)$. We further restricted our analyses to surgical cases that would be "acute care" using the variable "ATYPE" (Admission Type) and values equal to "Emergency Or (1)" or "Urgent or (2)". Following this restriction, we were left with 893,861 patients for analyses who represented adult patients that underwent acute care abdominal surgery.

\section{Study Variables}

Our study outcomes included 5 commonly used variables in surgical outcomes research. In-hospital mortality, any surgical complication, any medical complication were the 3 categorical outcomes with dichotomous responses (Yes vs. No). We identified surgical and medical complications using a list of ICD-9-CM diagnoses codes found in the published literature (Appendix 1) [19]. We did not count the total number of complications but rather analyzed the data for occurrence of a complication, regardless of the total count. Hospital length of stay (LOS), in days, and total hospital charges were the 2 continuous outcomes. We did not transform the data for our continuous measures and instead used the data in their original form.

Our primary exposure was liver disease status. Study patients were assigned to 3 liver disease groups: no liver disease, non-cirrhotic liver disease, and cirrhotic liver disease. The assignment was based on the presence of the following of ICD9-CM diagnoses codes, in position 1 to 15: non-cirrhotic liver disease $(070.22,070.23,070.32,070.33,070.44,070.54,456.0$, 456.1, 456.20, 456.21, 571.0, 571.3, 571.40, 571.8, 571.9, 572.3, and 572.8) and cirrhotic liver disease (571.2, 571.5, and 571.6). Patients with both non-cirrhotic and cirrhotic liver disease codes were assigned to the cirrhotic liver disease group. Our second primary exposure group was type of surgery: laparoscopic or open surgery.

We used several patient-level characteristics and hospitallevel characteristics in our multivariable regression analyses. Patients' age $(<40,50-64,65-79, \geqq 80)$, gender, race (White, Black, Hispanic, Other, Unknown), type of insurance (Medicare, Medicaid, private, self-pay, other, no charge), and number of chronic comorbidities (none, one, two, three or more) from the list of 29 co-existing medical illnesses provided in the NIS database (excluding liver disease) were our patient-level variables and all of them were categorical in nature. Similarly, we had 4 categorical variables pertaining to the hospitals from which the patients were sampled. The hospital-level variables were hospital size (small, medium, large), region (Northeast, Midwest, South, and West), teaching vs non-teaching, and location (urban vs rural).

\section{Statistical Analyses}

All analyses were performed using SAS statistical software version 9.4 (SAS Institute, Inc.). Descriptive statistics were used to describe the patient and hospital characteristics for overall study population and by type of surgery and liver disease status. Our study outcomes were also presented for the overall study population and by type of surgery and liver disease status. Categorical variables were described using counts and proportions. Means (Standard Deviations) and medians (interquartile ranges) were used to describe continuous variables.

Data in the NIS database is hierarchical in nature i.e. patients are nested (or clustered) within hospitals and hence study 
outcomes cannot be assessed using conventional regression models which assume independence of observations. To account for the clustered nature of study design we fit generalized linear models using PROC GENMOD. REPEATED statement with SUBJECT=HOSPID was used to invoke generalized estimating equations (GEE) methods to account for the clustering of patients within hospitals and to allow computation of robust standard errors. When modelling for categorical outcomes (inhospital mortality, any surgical, and any medical complication), we assumed a binary distribution with logit-link function and exchangeable working correlation structure. We modelled the continuous outcomes (hospital length of stay and total charges) in their original form, without any transformation, using loglink and exchangeable working correlation structure. Negative binomial and gamma distributions were used to model hospital length of stay and total charges, respectively.

We fit 5 sequential models for each of our study outcomes. Our model 1, unadjusted analyses, included only liver disease status as explanatory variable. We re-ran model 1 with type of surgery alone. Sequential multivariable regression models were fit with incremental addition of explanatory variables in the following order- Model 2 (liver disease status and type of surgery); Model 3 (model2 variables + patient characteristics [age, gender, race, type of insurance, and number of comorbidities]; Model 4 (model 3 variables plus hospital characteristics [region, size, location, and teaching status]; Model 5 (model 4 variables plus interaction term between liver disease status and type of surgery). For all outcomes our saturated models had the lowest Quasi-likelihood under the Independence Model Criterion (QIC) scores. LSMEANS statement was used to obtain odds ratios for variables in the interaction term. Exponentiation of parameter estimates obtained when using LSMEANS statement along with DIFF option provided adjusted odds ratios (for categorical outcomes) and adjusted ratios (for continuous outcomes) for the other variables in the model. We presented point-estimates with $95 \%$ confidence intervals. Given our large sample size we used a conservative cutoff of $p$-value $<0.01$ to infer statistical significance.

\section{Results}

\section{Study Cohort}

In all a total of 893,681 adult patients were identified as having undergone acute care abdominal surgery between 2006 and 2010 (Table 1). Some form of liver disease was present in 26, 453 patients. Among those with some form of liver disease, non-cirrhotic liver disease $(1.69 \%)$ was slightly more common than cirrhotic (1.27\%). Almost half of the acute care abdominal surgeries performed were laparoscopic surgeries. Close to $40 \%$ of the patients were between ages 40 and 64; this proportion was highest (61.3\%) among those with cirrhotic liver disease. Females comprised of 6 out of 10 study patients; but only 4 out of 10 in those with cirrhotic liver disease. Whites (56\%) and Unknown Race (19.8\%) were the two most frequent race categories. Similar proportion of patients were covered by private insurance (41.3\%) and Medicare/Medicaid (44.5\%); private insurance coverage was lowest for patients with cirrhosis
(28.7\%). Thirty-one percent of patients didn't have any chronic comorbidities; whereas almost all patients with cirrhotic liver disease $(92.9 \%)$ had one or more chronic comorbidities. Patients undergoing laparoscopic surgery were relatively younger, more female, had higher rates of private insurance coverage, and fewer comorbidities. Majority of the hospitals were urban (85.8\%), large $(62.1 \%)$, from the South $(44.2 \%)$. Hospitals where patients with cirrhotic liver disease received surgical care, compared to those without liver disease were larger, more urban, and more likely to be academic centers (or teaching hospitals).

\section{Study Outcomes}

In-hospital mortality was almost thrice in those with cirrhotic liver disease compared to the no liver disease group (9.3\% vs. $3.0 \%)$. There was more than a 10 -fold increase in In-hospital mortality in patients receiving open surgery compared to laparoscopic surgery (5.7\% vs $0.5 \%)$. Patients experienced at least one medical complication more frequently than a surgical complication $(22.8 \%$ vs. $8.1 \%)$. As for In-hospital mortality these rates were higher for those undergoing open surgery and in patients with cirrhotic liver disease (Table 2). The median hospital length of stay was 4 days for the overall study population. At least, half of the patients undergoing open surgery and those with cirrhotic liver disease stayed in the hospital for 7 or more days. Median length of stay for patients undergoing laparoscopic surgery was less than half that of those undergoing open surgery ( 3 vs. 7 days). The median total charge of $\$ 51,016$ for patients with cirrhotic liver disease was highest compared to other patient groups and was almost $\$ 20,000$ more than the overall median total charge. Median total charges for patients undergoing open surgery were more than twice than those for laparoscopic surgery ( $\$ 74,222$ vs. $\$ 33,394)$.

\section{Unadjusted Regression Results}

We ran two sets of unadjusted regression analyses, each with either liver disease status or type of surgery variable alone as the explanatory variable in the model (Data not shown).Results from our unadjusted regression analyses showed that having cirrhotic liver disease was associated with significantly higher risk for poor surgical outcomes when compared with having noncirrhotic or no liver disease. Increase in risk was observed for all outcomes, with the highest for in-hospital mortality ( $\sim 2.5$ times) and less moderate for hospital length of stay $(\sim 30 \%$ higher). Surgical outcomes didn't differ significantly between patients without liver disease and with non-cirrhotic liver disease, except for $11 \%$ increase in total hospital charges for non-cirrhotic liver disease group. Laparoscopic surgery was associated with marked reduction in mortality $(92 \%)$ and surgical complications $(86 \%)$ compared to open surgery. Moderate yet still very significant reduction $(\sim 60 \%)$ was seen for medical complications, length of stay and total charges.

\section{Adjusted regression results}

Table 3 illustrates the results from our adjusted multivariable logistic regression models. After adjusting for patient and hospital characteristics we observed significantly increased risk 


\begin{tabular}{|c|c|c|c|c|c|c|}
\hline \multirow[b]{2}{*}{ Characteristic } & \multirow{2}{*}{$\begin{array}{c}\text { Overall } \\
(\mathrm{N}=893,681)\end{array}$} & \multicolumn{2}{|c|}{ Type of Surgery } & \multicolumn{3}{|c|}{ Liver disease status } \\
\hline & & $\begin{array}{c}\text { Laparoscopic } \\
(\mathrm{N}=\mathbf{4 4 4 , 8 5 7 )}\end{array}$ & $\begin{array}{c}\text { Open } \\
(\mathrm{N}=448,824)\end{array}$ & $\begin{array}{c}\text { No } \quad \text { Liver } \\
\text { Disease } \\
(\mathrm{N}=\mathbf{8 6 7 , 2 0 0 )}\end{array}$ & $\begin{array}{c}\text { Non-Cirrhotic } \\
\text { Liver Disease } \\
(\mathrm{N}=15,138)\end{array}$ & $\begin{array}{c}\text { Cirrhotic Liver } \\
\text { Disease } \\
(\mathrm{N}=11,343)\end{array}$ \\
\hline \multicolumn{7}{|c|}{ Age categories } \\
\hline$<40$ & 29.6 & 38.5 & 20.8 & 30.1 & 22.9 & 5.0 \\
\hline $40-64$ & 38.6 & 38.1 & 39.1 & 38.0 & 57.9 & 61.3 \\
\hline $65-79$ & 20.4 & 15.6 & 25.1 & 20.4 & 15.5 & 25.5 \\
\hline$\geq 80$ & 11.4 & 7.8 & 15.0 & 11.6 & 3.7 & 8.3 \\
\hline Female & 58.5 & 61.8 & 55.2 & 58.8 & 53.9 & 40.7 \\
\hline \multicolumn{7}{|c|}{ Race } \\
\hline Black & 9.6 & 8.0 & 11.2 & 9.6 & 10.3 & 8.6 \\
\hline Hispanic & 9.8 & 12.3 & 7.4 & 9.8 & 11.3 & 10.5 \\
\hline Other & 4.8 & 5.3 & 4.3 & 4.8 & 5.0 & 4.5 \\
\hline Unknown & 19.8 & 18.6 & 21.1 & 19.9 & 16.4 & 19.6 \\
\hline White & 56.0 & 55.8 & 56.1 & 56.0 & 57.0 & 56.8 \\
\hline \multicolumn{7}{|c|}{ Type of Insurance } \\
\hline Medicare & 33.4 & 24.8 & 41.9 & 33.4 & 26.1 & 42.5 \\
\hline Medicaid & 11.1 & 11.6 & 10.5 & 11.0 & 14.0 & 15.8 \\
\hline Private & 41.3 & 47.2 & 35.6 & 41.4 & 44.4 & 28.7 \\
\hline Self-pay & 9.2 & 11.0 & 7.5 & 9.2 & 9.8 & 7.5 \\
\hline No Charge & 1.1 & 1.4 & 0.9 & 1.1 & 1.4 & 0.9 \\
\hline Other & 3.6 & 3.8 & 3.4 & 3.6 & 4.1 & 4.4 \\
\hline \multicolumn{7}{|c|}{ Chronic Comorbidities } \\
\hline None & 30.9 & 38.8 & 23.0 & 31.5 & 14.2 & 7.1 \\
\hline One & 22.5 & 24.3 & 20.8 & 22.6 & 22.4 & 15.6 \\
\hline Two & 18.3 & 16.7 & 19.9 & 18.2 & 23.4 & 22.9 \\
\hline Three or more & 28.3 & 20.2 & 36.4 & 27.8 & 40.0 & 54.4 \\
\hline \multicolumn{7}{|c|}{ Hospital Region } \\
\hline Northeast & 21.5 & 20.7 & 22.2 & 21.6 & 16.8 & 19.1 \\
\hline Midwest & 22.9 & 21.7 & 24.7 & 23.0 & 20.2 & 21.8 \\
\hline South & 44.2 & 45.2 & 43.3 & 44.1 & 48.5 & 45.9 \\
\hline West & 11.4 & 12.4 & 10.3 & 11.3 & 14.5 & 13.2 \\
\hline \multicolumn{7}{|c|}{ Hospital Bed Size } \\
\hline Small & 12.0 & 12.0 & 11.9 & 12.1 & 10.1 & 9.3 \\
\hline Medium & 24.9 & 25.6 & 24.1 & 24.9 & 23.7 & 22.0 \\
\hline Large & 62.1 & 61.4 & 62.8 & 62.0 & 64.6 & 67.7 \\
\hline Urban Hospital & 85.8 & 86.4 & 85.1 & 85.7 & 89.3 & 88.2 \\
\hline Teaching Hospital & 43.1 & 40.1 & 46.0 & 43.0 & 42.7 & 51.3 \\
\hline
\end{tabular}

for the three categorical outcomes (in-hospital death, any surgical complication, and any medical complication) in cirrhotic patients compared those with no liver disease. Results from same model, showed that laparoscopic surgery was protective, over open surgery, for the 3 categorical outcomes. For example, we found an $87 \%$ lower odds for in-hospital mortality among patients undergoing laparoscopic compared to open surgery. Of note, male gender, increasing age, Medicare/Medicaid status, and having more than one chronic comorbidity were significantly predictive of all three outcomes. Having increasing comorbidities had a much more profound predictive effect on surgical complications than the other two outcomes. Interestingly, hospital teaching 
Table 2: Study outcomes by type of surgery and liver disease status

\begin{tabular}{|c|c|c|c|c|c|c|}
\hline \multirow[b]{2}{*}{ Study Outcomes } & \multirow{2}{*}{$\begin{array}{c}\text { Overall } \\
(\mathrm{N}=\mathbf{8 9 3}, 681)\end{array}$} & \multicolumn{2}{|c|}{ Type of Surgery } & \multicolumn{3}{|c|}{ Liver disease status } \\
\hline & & $\begin{array}{l}\text { Laparoscopic } \\
(\mathrm{N}=444,857)\end{array}$ & $\begin{array}{c}\text { Open } \\
(\mathrm{N}=448,824)\end{array}$ & $\begin{array}{c}\text { No } \quad \text { Liver } \\
\text { Disease } \\
(\mathrm{N}=\mathbf{8 6 7 , 2 0 0 )}\end{array}$ & $\begin{array}{c}\text { Non-Cirrhotic } \\
\text { Liver Disease } \\
(\mathrm{N}=15,138)\end{array}$ & $\begin{array}{c}\text { Cirrhotic Liver } \\
\text { Disease } \\
(\mathrm{N}=11,343)\end{array}$ \\
\hline & \multicolumn{6}{|c|}{ Categorical Outcomes (in \%) } \\
\hline In-hospital death & 3.1 & 0.5 & 5.7 & 3.0 & 2.7 & 9.3 \\
\hline $\begin{array}{l}\text { Any surgical } \\
\text { complication }\end{array}$ & 8.1 & 2.3 & 13.9 & 8.1 & 7.4 & 13.2 \\
\hline \multirow[t]{2}{*}{$\begin{array}{l}\text { Any medical } \\
\text { complication }\end{array}$} & 22.8 & 14.4 & 31.1 & 22.6 & 21.7 & 39.1 \\
\hline & \multicolumn{6}{|c|}{ Continuous Outcomes } \\
\hline \multicolumn{7}{|l|}{$\begin{array}{c}\text { Hospital length of stay } \\
\text { (in days) }\end{array}$} \\
\hline Mean (SD) & $7.3(10.1)$ & $4.0(4.9)$ & $10.6(12.5)$ & $7.2(10.0)$ & $7.3(9.7)$ & $10.9(13.1)$ \\
\hline (P25-P75) & $4(2-9)$ & $3(2-5)$ & $7(4-13)$ & $4(2-9)$ & $5(3-8)$ & $7(4-13)$ \\
\hline \multicolumn{7}{|l|}{ Hospital charges (in \$) } \\
\hline Mean (SD) & $53,878(81,022)$ & $33,394(37,264)$ & $74,222(104,281)$ & $53,190(79,705)$ & $61,733(97,208)$ & $96,005(130,438)$ \\
\hline Median $\quad$ (P25-P75) & $\begin{array}{c}30,449 \\
(18,831-55,628)\end{array}$ & $\begin{array}{c}24,724 \\
(16,989-37,871)\end{array}$ & $\begin{array}{c}41,891 \\
(22,583-81,853)\end{array}$ & $\begin{array}{c}30,178 \\
(18,694-5,5038)\end{array}$ & $\begin{array}{c}36,326 \\
(22,945-61,263)\end{array}$ & $\begin{array}{c}51,016 \\
(28,368-106,001)\end{array}$ \\
\hline
\end{tabular}

Table 3: Adjusted odds ratios (with $\mathbf{9 5 \%}$ confidence intervals) for in-hospital mortality, surgical, and medical complications from multivariable regression models*

\begin{tabular}{|c|c|c|c|c|c|c|}
\hline & In-hospital death & & Any Surgical Complication & & $\begin{array}{l}\text { Any Medical } \\
\text { Complication }\end{array}$ & \\
\hline \multicolumn{7}{|c|}{ Liver disease status } \\
\hline Cirrhotic & $2.68(2.47-2.91)$ & $<.0001$ & $1.26(1.16-1.36)$ & $<.0001$ & $1.43(1.36-1.51)$ & $<.0001$ \\
\hline Non-Cirrhotic & $1.41(1.28-1.56)$ & $<.0001$ & $1.04(0.98-1.12)$ & 0.1984 & $1.00(0.95-1.05)$ & 0.9852 \\
\hline No liver disease & REF & . & REF & . & REF & . \\
\hline \multicolumn{7}{|l|}{ Type of surgery } \\
\hline Laparoscopic & $0.13(0.13-0.14)$ & $<.0001$ & $0.18(0.17-0.18)$ & $<.0001$ & $0.58(0.57-0.59)$ & $<.0001$ \\
\hline Open & REF & . & REF & . & REF & . \\
\hline \multicolumn{7}{|l|}{ Age categories } \\
\hline$<40$ & REF & . & REF & . & REF & . \\
\hline $40-64$ & $2.25(2.09-2.42)$ & $<.0001$ & $1.46(1.42-1.50)$ & $<.0001$ & $1.69(1.65-1.74)$ & $<.0001$ \\
\hline $65-79$ & $4.27(3.92-4.66)$ & $<.0001$ & $1.32(1.27-1.37)$ & $<.0001$ & $3.31(3.20-3.43)$ & $<.0001$ \\
\hline$\geq 80$ & $6.87(6.28-7.51)$ & $<.0001$ & $1.10(1.05-1.14)$ & $<.0001$ & 6.35 (6.12-6.59) & $<.0001$ \\
\hline \multicolumn{7}{|l|}{ Gender } \\
\hline Male & $1.22(1.19-1.26)$ & $<.0001$ & 1.07 (1.05-1.09) & $<.0001$ & $1.16(1.15-1.18)$ & $<.0001$ \\
\hline Female & REF & . & REF & . & REF & . \\
\hline \multicolumn{7}{|l|}{ Race } \\
\hline Black & $0.99(0.94-1.04)$ & 0.6232 & $0.98(0.95-1.02)$ & 0.307 & 1.07 (1.04-1.09) & $<.0001$ \\
\hline Hispanic & $0.85(0.79-0.90)$ & $<.0001$ & $0.92(0.88-0.96)$ & $<.0001$ & $0.85(0.82-0.87)$ & $<.0001$ \\
\hline Other & $1.03(0.96-1.11)$ & 0.3798 & $1.03(0.98-1.07)$ & 0.2863 & $0.90(0.87-0.93)$ & $<.0001$ \\
\hline Unknown & $1.12(1.06-1.18)$ & $<.0001$ & $0.94(0.90-0.98)$ & 0.0023 & $1.02(0.99-1.05)$ & 0.1336 \\
\hline White & REF & . & REF & . & REF & . \\
\hline \multicolumn{7}{|c|}{ Type of insurance } \\
\hline Medicaid & $1.44(1.36-1.52)$ & $<.0001$ & 1.04 (1.01-1.08) & 0.0161 & 1.45 (1.41-1.49) & $<.0001$ \\
\hline Medicare & $1.48(1.41-1.55)$ & $<.0001$ & $1.16(1.13-1.20)$ & $<.0001$ & $1.56(1.52-1.59)$ & $<.0001$ \\
\hline No Charge & $0.89(0.70-1.13)$ & 0.3401 & $0.83(0.74-0.93)$ & 0.0011 & $1.02(0.95-1.10)$ & 0.5722 \\
\hline Other & $1.23(1.10-1.37)$ & 0.0002 & $1.04(0.99-1.10)$ & 0.123 & $1.13(1.08-1.18)$ & $<.0001$ \\
\hline Self-Pay & $1.24(1.14-1.34)$ & $<.0001$ & $0.78(0.75-0.81)$ & $<.0001$ & $1.03(1.00-1.07)$ & 0.0318 \\
\hline
\end{tabular}




\begin{tabular}{|c|c|c|c|c|c|c|}
\hline Private & REF & . & REF & . & REF & . \\
\hline \multicolumn{7}{|c|}{ No of chronic comorbidities } \\
\hline None & REF & . & REF & . & REF & . \\
\hline One & $1.59(1.49-1.70)$ & $<.0001$ & $1.41(1.37-1.46)$ & $<.0001$ & $1.97(1.92-2.02)$ & $<.0001$ \\
\hline Two & $1.98(1.83-2.15)$ & $<.0001$ & $1.54(1.48-1.59)$ & $<.0001$ & $3.02(2.92-3.12)$ & $<.0001$ \\
\hline Three or more & $2.91(2.66-3.18)$ & $<.0001$ & $1.68(1.61-1.76)$ & $<.0001$ & $6.82(6.52-7.13)$ & $<.0001$ \\
\hline \multicolumn{7}{|c|}{ Hospital bed size } \\
\hline Small & $0.72(0.67-0.77)$ & $<.0001$ & $0.87(0.82-0.92)$ & $<.0001$ & $0.79(0.76-0.83)$ & $<.0001$ \\
\hline Medium & $0.89(0.84-0.94)$ & $<.0001$ & $0.91(0.87-0.95)$ & $<.0001$ & $0.89(0.86-0.92)$ & $<.0001$ \\
\hline Large & REF &. & REF & . & REF & . \\
\hline \multicolumn{7}{|c|}{ Hospital location } \\
\hline Rural & $0.84(0.79-0.9)$ & $<.0001$ & $0.91(0.86-0.96)$ & 0.0002 & $0.77(0.74-0.79)$ & $<.0001$ \\
\hline Urban & REF & . & REF & . & REF & . \\
\hline \multicolumn{7}{|l|}{ Hospital region } \\
\hline Northeast & $1.15(1.05-1.26)$ & 0.0018 & $0.77(0.71-0.84)$ & $<.0001$ & $0.99(0.93-1.05)$ & 0.7225 \\
\hline Midwest & $0.90(0.82-0.98)$ & 0.0141 & $0.81(0.75-0.87)$ & $<.0001$ & $1.00(0.95-1.06)$ & 0.9505 \\
\hline South & $1.11(1.03-1.21)$ & 0.0102 & $0.78(0.73-0.83)$ & $<.0001$ & $1.03(0.97-1.08)$ & 0.3484 \\
\hline West & REF &. & REF & . & REF & . \\
\hline \multicolumn{7}{|c|}{ Teaching Hospital } \\
\hline No & $0.78(0.73-0.82)$ & $<.0001$ & $0.77(0.74-0.81)$ & $<.0001$ & $0.87(0.85-0.9)$ & $<.0001$ \\
\hline Yes & REF & . & REF & . & REF & . \\
\hline
\end{tabular}

status, rural location, small and medium hospital size, Hispanic race, and laparoscopic surgery type were protective for all outcomes.

Table 4 illustrates the results from our adjusted multivariable generalized linear models for the two continuous outcomes (hospital length of stay and total charges).As expected, patients with no liver disease who underwent acute care surgery had a shorter length of stay and lower hospital charges than cirrhotic and non-cirrhotic liver disease patients; cirrhotic liver disease portended the longest length of stay and highest hospital charges compared to the other two. In this adjusted model, laparoscopic surgery, younger age, Hispanic race, small and medium hospital size, rural hospital location, and non-teaching hospital status were predictive of lower hospital length of stay and total charges. In contrast, male gender, and Medicare and Medicaid insurance status were related to higher length of stay and total charges.

\section{Study Outcomes by Liver Disease Status within type of} Surgery

Apart from the main effects of liver disease and type of surgery, we further examined association between each variable and study outcomes when conditioned on the other using an interaction-term between liver disease and type of surgery in our models (Table 5). Among patients who underwent laparoscopic surgery, the findings were: a) when compared to patients without liver disease, cirrhotic patients experienced increased risk for poor outcomes, ranging from 31 to $194 \%$ across the 5 study outcomes; b) the same was true for cirrhotic versus non-cirrhotic liver disease groups (increase in risk by 11 to $161 \%$ ); c) the ratio for hospital length of stay and total charges were 3 to $7 \%$ higher for those with non-cirrhotic liver disease over those without any liver disease, despite no significant differences in risk for in-hospital mortality and complications. When studying patients who underwent open surgery, we found: a) similar increased risk of poor outcomes, between 24 to $164 \%$, amongst patients with cirrhosis over those without any liver disease; b) when compared with non-cirrhotic liver disease patients, patients with cirrhosis had higher mortality and medical complication risk and higher hospital charges; c) patients with non-cirrhotic liver disease were associated with higher in-hospital mortality, hospital length of stay and total charges compared to those without any liver disease.

\section{Study Outcomes by type of surgery within each liver disease status}

Within each of the 3 liver disease groups, we found a consistent relationship between type of surgery and study outcomes (Table 6). Laparoscopic surgery was associated with reduced risk for poor clinical outcomes compared to open surgery. The reduction in risk was larger for outcomes such as in-hospital mortality and surgical complications (by 90 to 81\%). Less moderate yet still significant reduction (by $54 \%$ to $41 \%$ ) was observed for medical complications, length of stay, and total charges.

\section{Discussion}

Currently, no major study has done a comparative evaluation of laparoscopic versus open surgical technique in acute care general surgery patients with a focus on liver disease status. Apart from being the first one to perform this analyses, it also differs from prior studies in that we use a large nationally representative sample to examine differences in acute care surgical outcomes in patients with cirrhosis and non-cirrhotic liver disease.

Our study has a number of important findings. First, inhospital mortality was higher amongst patients with cirrhotic 
Table 4: Adjusted ratios (with $95 \%$ confidence intervals) for relative hospital length of stay and total charges from multivariable regression models*

\begin{tabular}{|c|c|c|c|c|}
\hline & Hospital Length of Stay & & Hospital Total Charges & \\
\hline \multicolumn{5}{|c|}{ Liver disease status } \\
\hline Cirrhotic & $1.16(1.13-1.20)$ & $<.0001$ & $1.32(1.24-1.40)$ & $<.0001$ \\
\hline Non-Cirrhotic & $1.08(1.06-1.10)$ & $<.0001$ & $1.11(1.07-1.14)$ & $<.0001$ \\
\hline No liver disease & REF & . & REF & . \\
\hline \multicolumn{5}{|c|}{ Type of surgery } \\
\hline Laparoscopic & $0.47(0.47-0.48)$ & $<.0001$ & $0.56(0.55-0.57)$ & $<.0001$ \\
\hline Open & REF & . & REF & . \\
\hline \multicolumn{5}{|c|}{ Age categories } \\
\hline$<40$ & $1.27(1.26-1.28)$ & $<.0001$ & $1.18(1.17-1.19)$ & $<.0001$ \\
\hline $40-64$ & $1.37(1.35-1.40)$ & $<.0001$ & $1.27(1.25-1.29)$ & $<.0001$ \\
\hline $65-79$ & $1.46(1.44-1.49)$ & $<.0001$ & $1.27(1.25-1.30)$ & $<.0001$ \\
\hline$\geq 80$ & REF & . & REF & . \\
\hline \multicolumn{5}{|c|}{ Gender } \\
\hline Male & $1.08(1.07-1.09)$ & $<.0001$ & $1.12(1.11-1.13)$ & $<.0001$ \\
\hline Female & REF & . & REF & . \\
\hline \multicolumn{5}{|l|}{ Race } \\
\hline Black & $1.08(1.07-1.1)$ & $<.0001$ & $1.03(1.01-1.04)$ & 0.0003 \\
\hline Hispanic & $0.97(0.96-0.98)$ & $<.0001$ & $0.95(0.93-0.96)$ & $<.0001$ \\
\hline Other & $1.00(0.99-1.02)$ & 0.5729 & $1.00(0.98-1.01)$ & 0.6863 \\
\hline Unknown & $1.05(1.03-1.06)$ & $<.0001$ & $0.96(0.94-0.99)$ & 0.0016 \\
\hline White & REF & . & REF & . \\
\hline \multicolumn{5}{|c|}{ Type of insurance } \\
\hline Medicaid & $1.23(1.22-1.25)$ & $<.0001$ & $1.13(1.11-1.14)$ & $<.0001$ \\
\hline Medicare & $1.20(1.19-1.22)$ & $<.0001$ & $1.16(1.14-1.17)$ & $<.0001$ \\
\hline No Charge & $1.06(1.03-1.10)$ & 0.0005 & $1.01(0.96-1.05)$ & 0.8069 \\
\hline Other & $1.09(1.07-1.11)$ & $<.0001$ & $1.06(1.03-1.08)$ & $<.0001$ \\
\hline Self-Pay & $1.02(1.01-1.03)$ & 0.0012 & $1.00(0.99-1.01)$ & 0.8254 \\
\hline Private & REF & . & REF & . \\
\hline \multicolumn{5}{|c|}{ No of chronic comorbidities } \\
\hline None & $2.11(2.06-2.16)$ & $<.0001$ & $1.87(1.83-1.92)$ & $<.0001$ \\
\hline One & $1.35(1.34-1.37)$ & $<.0001$ & $1.25(1.23-1.26)$ & $<.0001$ \\
\hline Two & $1.61(1.59-1.64)$ & $<.0001$ & $1.44(1.42-1.47)$ & $<.0001$ \\
\hline Three or more & REF & . & REF & . \\
\hline \multicolumn{5}{|c|}{ Hospital bed size } \\
\hline Small & $0.90(0.88-0.92)$ & $<.0001$ & $0.90(0.86-0.94)$ & $<.0001$ \\
\hline Medium & $0.84(0.81-0.86)$ & $<.0001$ & $0.78(0.75-0.83)$ & $<.0001$ \\
\hline Large & REF & . & REF & . \\
\hline \multicolumn{5}{|l|}{ Hospital location } \\
\hline Rural & $0.83(0.81-0.84)$ & $<.0001$ & $0.65(0.63-0.68)$ & $<.0001$ \\
\hline Urban & REF & . & $1(1-1)$ & . \\
\hline \multicolumn{5}{|c|}{ Hospital region } \\
\hline Northeast & $1.12(1.08-1.15)$ & $<.0001$ & $0.91(0.84-0.98)$ & 0.0119 \\
\hline Midwest & $0.94(0.91-0.97)$ & 0.0001 & $0.84(0.79-0.88)$ & $<.0001$ \\
\hline South & $1.06(1.02-1.09)$ & 0.0005 & $1.03(0.98-1.09)$ & 0.2451 \\
\hline West & REF & . & REF & . \\
\hline \multicolumn{5}{|c|}{ Teaching Hospital } \\
\hline No & $0.81(0.79-0.83)$ & $<.0001$ & $0.82(0.78-0.87)$ & $<.0001$ \\
\hline Yes & REF & . & REF & . \\
\hline
\end{tabular}


Table 5: Study outcomes by liver disease status within type of surgery*\#

\begin{tabular}{|c|c|c|c|c|}
\hline & \multicolumn{2}{|c|}{ Laparoscopic Surgery } & \multicolumn{2}{|c|}{ Open Surgery } \\
\hline \multicolumn{5}{|c|}{ Categorical Outcomes } \\
\hline & $\begin{array}{c}\text { Adjusted Odds Ratios } \\
\text { (95\% CI) }\end{array}$ & p-value & $\begin{array}{c}\text { Adjusted Odds Ratios } \\
\text { (95\% CI) }\end{array}$ & p-value \\
\hline \multicolumn{5}{|c|}{ In-hospital mortality } \\
\hline Cirrhotic vs No liver disease & $2.94(2.47-3.51)$ & $<.0001$ & $2.64(2.43-2.88)$ & $<.0001$ \\
\hline Non-Cirrhotic vs No liver disease & $1.13(0.90-1.42)$ & 0.3003 & $1.48(1.33-1.65)$ & $<.0001$ \\
\hline Cirrhotic vs Non-Cirrhotic & $2.61(1.96-3.48)$ & $<.0001$ & $1.79(1.57-2.04)$ & $<.0001$ \\
\hline \multicolumn{5}{|c|}{ Any Surgical Complication } \\
\hline Cirrhotic vs No liver disease & $1.34(1.16-1.54)$ & $<.0001$ & $1.24(1.13-1.36)$ & $<.0001$ \\
\hline Non-Cirrhotic vs No liver disease & $0.95(0.84-1.07)$ & 0.374 & $1.08(1.00-1.18)$ & 0.0511 \\
\hline Cirrhotic vs Non-Cirrhotic & $1.41(1.17-1.69)$ & 0.0002 & $1.14(1.02-1.28)$ & 0.0227 \\
\hline \multicolumn{5}{|c|}{ Any Medical Complication } \\
\hline Cirrhotic vs No liver disease & $1.31(1.22-1.40)$ & $<.0001$ & $1.53(1.42-1.64)$ & $<.0001$ \\
\hline Non-Cirrhotic vs No liver disease & $0.93(0.88-0.99)$ & 0.0251 & $1.09(1.01-1.17)$ & 0.0215 \\
\hline Cirrhotic vs Non-Cirrhotic & $1.40(1.28-1.53)$ & $<.0001$ & $1.40(1.27-1.55)$ & $<.0001$ \\
\hline \multicolumn{5}{|c|}{ Hospital Length of Stay } \\
\hline Cirrhotic vs No liver disease & $1.25(1.21-1.28)$ & $<.0001$ & $1.11(1.06-1.16)$ & $<.0001$ \\
\hline Non-Cirrhotic vs No liver disease & 1.07 (1.05-1.09) & $<.0001$ & $1.09(1.05-1.13)$ & $<.0001$ \\
\hline Cirrhotic vs Non-Cirrhotic & $1.16(1.13-1.20)$ & $<.0001$ & $1.02(0.96-1.07)$ & 0.5593 \\
\hline \multicolumn{5}{|c|}{ Hospital Total Charges } \\
\hline Cirrhotic vs No liver disease & $1.14(1.09-1.20)$ & $<.0001$ & $1.46(1.35-1.58)$ & $<.0001$ \\
\hline Non-Cirrhotic vs No liver disease & $1.03(1.00-1.04)$ & 0.0026 & $1.26(1.18-1.35)$ & $<.0001$ \\
\hline Cirrhotic vs Non-Cirrhotic & $1.11(1.06-1.17)$ & $<.0001$ & $1.16(1.06-1.26)$ & 0.0011 \\
\hline
\end{tabular}

*Model includes: liver disease status, type or surgery, interaction between liver disease and type of surgery, age, gender, race, insurance, comorbidities, hospital region, size, location and teaching status. $\mathrm{CI}=$ Confidence Interval.

\#Conservative $\mathrm{p}$-value of $<0.01$ was used to determine significant associations given the large sample size.

and non-cirrhotic disease groups compared those without liver disease; second, in acute care surgery setting, laparoscopic surgery was associated with better surgical outcomes regardless of the type of liver disease; third, when undergoing laparoscopic surgery patients with cirrhosis are more likely to be associated with poorer surgical outcomes compared to non-cirrhotic and no liver disease patients; fourth; similar excess risk is associated with cirrhotic patients undergoing open surgery; and fifth, patients with non-cirrhotic liver disease who undergo open surgery have increased in-hospital mortality, length of stay and total charges compared to those without liver disease.

Our results are consistent with a number of studies in the literature that compare laparoscopic and open surgical technique in acute care general surgical patients. Tsugawa, et al show that laparoscopic appendectomy resulted in fewer postoperative complications, improved postoperative pain, and lower length of stay in cirrhotic patients, as compared to open appendectomy [20]. In their prospective, randomized study, Hamad, et al concluded that laparoscopic cholecystectomy was comparable to the open approach across all measured outcomes, however, with shorter length of stay and less pain, in Childs-Pugh class A and B cirrhotics [21]. They suggest that the laparoscopic cholecystectomy is the preferred option in this class of patients with cirrhosis. Poggio, et al came to the same conclusion regarding laparoscopic surgery in compensated cirrhotics in a retrospective cohort study of 50 cholecystectomy patients [22].

The overall role of laparoscopy in emergent and urgent surgery is expanding at a rapid rate. Aside from the classic indications of appendicitis and acute cholecystitis, laparoscopy has gained traction in the treatment of diverticulitis, adhesive intestinal obstruction, perforation and/or bleeding from gastroduodenal ulcer disease, diagnosis of abdominal extremis, and even trauma. Laparoscopic peritoneal lavage has proven to be an effective emergent treatment for perforated diverticulitis with a low conversion and high efficacy rate [23-25]. Furthermore, Ming-Zhe et al showed that laparoscopic adhesiolysis for small bowel obstruction was not inferior with respect to the number of iatrogenic enterotomies, surgical site infection, pulmonary complications, or overall mortality, and this has been supported by numerous studies [26-29]. Finally, two important randomized studies, albeit with small populations, have shown the non-inferiority of laparoscopic management of perforated gastroduodenal ulcer disease [30,31]. Our data is consistent with 


\begin{tabular}{|c|c|c|c|c|c|c|}
\hline & \multicolumn{2}{|c|}{ No liver disease } & \multicolumn{2}{|c|}{ Non-Cirrhotic liver disease } & \multicolumn{2}{|c|}{ Cirrhotic liver disease } \\
\hline \multicolumn{7}{|c|}{ Categorical Outcomes } \\
\hline & $\begin{array}{c}\text { Adjusted Odds Ratios } \\
(95 \% \mathrm{CI})\end{array}$ & p-value & $\begin{array}{l}\text { Adjusted Odds } \\
\text { Ratios }(95 \% \mathrm{CI})\end{array}$ & p-value & $\begin{array}{c}\text { Adjusted Odds } \\
\text { Ratios (95\% CI) }\end{array}$ & p-value \\
\hline \multicolumn{7}{|c|}{ In-hospital mortality } \\
\hline Laparoscopic vs Open & $0.13(0.13-0.14)$ & $<.0001$ & $0.10(0.08-0.13)$ & $<.0001$ & $0.15(0.12-0.18)$ & $<.0001$ \\
\hline \multicolumn{7}{|c|}{ Any Surgical Complication } \\
\hline Laparoscopic vs Open & $0.18(0.17-0.18)$ & $<.0001$ & $0.16(0.14-0.18)$ & $<.0001$ & $0.19(0.16-0.23)$ & $<.0001$ \\
\hline \multicolumn{7}{|c|}{ Any Medical Complication } \\
\hline Laparoscopic vs Open & $0.59(0.58-0.60)$ & $<.0001$ & $0.50(0.46-0.55)$ & $<.0001$ & $0.50(0.45-0.55)$ & $<.0001$ \\
\hline \multicolumn{7}{|c|}{ Continuous Outcomes } \\
\hline & $\begin{array}{l}\text { Adjusted Ratios (95\% } \\
\text { CI) }\end{array}$ & p-value & $\begin{array}{l}\text { Adjusted Ratios }(95 \% \\
\text { CI) }\end{array}$ & p-value & $\begin{array}{l}\text { Adjusted Ratios } \\
\quad(95 \% \mathrm{CI})\end{array}$ & p-value \\
\hline \multicolumn{7}{|c|}{ Hospital Length of Stay } \\
\hline Laparoscopic vs Open & $0.47(0.47-0.48)$ & $<.0001$ & $0.46(0.44-0.49)$ & $<.0001$ & $0.53(0.51-0.56)$ & $<.0001$ \\
\hline \multicolumn{7}{|c|}{ Hospital Total Charges } \\
\hline Laparoscopic vs Open & $0.56(0.56-0.57)$ & $<.0001$ & $0.46(0.43-0.49)$ & $<.0001$ & $0.44(0.41-0.47)$ & $<.0001$ \\
\hline
\end{tabular}

*Model includes: liver disease status, type or surgery, interaction between liver disease and type of surgery, age, gender, race, insurance, comorbidities, hospital region, size, location and teaching status. $\mathrm{CI}=$ Confidence Interval.

\#Conservative p-value of $<0.01$ was used to determine significant associations given the large sample size.

the current literature which shows that emergent and urgent acute care general surgery in liver failure patients portends poorer outcomes. Andraus, et al showed that emergency surgery was a significant independent risk factor for higher mortality in hernia surgery patients with cirrhosis [32]. Short term (40\%) and long-term mortality (54\%) in abdominal operations in patients with cirrhosis is independently influenced by having an emergency operation. In a comprehensive systematic review by Goede, cirrhotic patients undergoing emergency surgery had a higher mortality compared to non-emergent surgical patients with an odds ratio of 2.4 [33]. Due to higher complications, longer length of stay, increased need for bowel resection, and increased operative time with emergency umbilical hernia repair, Choir et al advocate an elective approach whenever possible [34]. Lehnert showed in a retrospective analysis that patients with liver failure needing acute care surgery for bleeding or perforated peptic ulcers had a mortality that was doubled that of elective resection [35]. These studies are in line with the findings of our large cohort study with respect to the outcomes of acute care general surgery in the liver disease population.

The type of liver failure, its severity, and its sequelae can have a profound effect on outcomes after general surgery, however the literature is sparse in addressing this as it relates to acute care surgery [36]. Postoperative mortality was increased in patients who had liver cirrhosis with viral hepatitis or alcoholic liver disease, according to Lin, et al [37]. Indeed, outcomes after cardiac surgery in cirrhotics was clearly related to the Child's Pugh classification of the cirrhosis [38]. The liver plays an important role in the regulation of nutrition and metabolism; thus, it is not unexpected that liver disease patients may have poor nutrition. Garrison et al used a multivariate analysis to predict survival after abdominal surgery in patients with liver failure, and found that poor nutrition was a significant risk factor in this set of patients, with poor nutrition presumably as a sequelae of the liver failure itself [39]. Specific aspects of liver failure that may contribute to mortality in non-liver surgery are elusive, however, Rice, et al determined that international normalized ratio greater than 1.6 and encephalopathy independently predicted mortality in this population. Interestingly, in their study, Child's classification did not predict mortality [40].

There are several limitations to our study. Although we were able to study a large sample size, our study is limited in that it is not a randomized controlled trial. Our data lack granular information on occurrence of fatty liver disease, liver function tests, Child's Pugh scores, and Model of End-Stage Liver Disease (MELD) scores, rendering us unable to differentiate cohorts based on liver disease severity. The data set provides in-hospital data only, with no information available about prior liver disease history or surgery history, or follow-up after discharge. Some patients will undergo acute care surgical procedures (such as appendectomy) and be discharged within 24 hours, which then makes them outpatient status at many hospitals and thus may not be included in the NIS. If this were a substantial proportion of procedures, it would likely cause our analysis to overestimate the procedural complication rate and to underestimate the proportion of patients treated in this type of outpatient setting. Although NIS is a national database with wide reaching penetration, participation is still voluntary. Therefore, our conclusions may not be wholly applicable for every hospital or provider. Nevertheless, our study represents one of the larger series and may be broadly generalizable due to the cohort of hospitals that contribute to the NIS. 
Another universal drawback on the use of administrative databases that rely on ICD-9-CM codes is the lack of fidelity in ensuring an accurate diagnosis. Human errors, including but not limited to, under-reporting of an event or inaccuracy in coding or even miscoding, cannot be ruled out to impact our projected estimates. In addition, outcomes are reported for the duration of an inpatient stay; therefore, extrapolation of these data to reflect outcomes beyond this period must be done with caution. In addition, certain outcomes cannot be measured meaningfully within the inpatient time period. One example of an important long-term morbidity of hernia repair is recurrence, which occurs well beyond the inpatient time period and seems to occur progressively over time. Granular details such as the preoperative status of patients, certain symptoms in the perioperative period, use of certain medications that portend risk (e.g., anticoagulants), or symptom recurrence, among others, are not collected within this type of database, and therefore, these important factors are not taken into account in our study.

\section{Conclusion}

Our study statistical analysis on this restricted population has shown that laparoscopic techniques in acute care general surgery patients with liver disease may have a protective effect as compared to certain open procedure. As our study is limited due to the nature of the dataset and retrospective analysis, further prospective studies are needed to adequately address the best way to treat liver disease patients who need emergent and urgent abdominal surgery.

\section{Declaration}

\section{Ethical Approval}

Institutional Review Board approval (Protocol Number Pro20150002233) was obtained for this study.

\section{References}

1. Sheen I, Liaw Y. The prevalence and incidence of cholecystolithiasis in patients with chronic liver disease: a prospective study. Hepatology. 1989;9(4):538-40.

2. Nicholas P, Rinaudo PA, Conn HO. Increased incidence of cholelithiasis in Laennec's cirrhosis, a postmortem evaluation of pathogenesis. Gastroenterology. 1972;63(1):112-21.

3. Sonnenberg A. Concordant occurrence of gastric and hypertensive disease. Gastroenterology. 1988;95(1):42-8.

4. Belghiti J, Durand F. Abdominal wall hernias in the setting of cirrhosis. Semin Liver Dis. 1997;17(3):219-26.

5. Jackson FC, Christophersen EB, Peternel WW, Kirimli B. Preoperative management of patients with liver disease. Surg Clin North Am. 1968;48(4):907-30.

6. Propst A, Propst T, Zangerl G, Ofner D, Judmaier G, Vogel W. Prognosis and life expectancy in chronic liver disease. Dig Dis Sci. 1995;40(8):1805-15

7. Doberneck RC, Sterling WA Jr, Allison DC. Allison. Morbidity and mortality after operation in nonbleeding cirrhotic patients. Am J Surg. 1983;146(3):306-9.

8. Ziser A, Plevak DJ, Wiesner RH, Rakela J, Offord KP, Brown DL
Morbidity and mortality in cirrhotic patients undergoing anesthesia and surgery. Anesthesiology. 1999;90(1):42-53.

9. del Olmo JA, Flor-Lorente B, Flor-Civera B, Rodriguez F, Serra MA, Escudero A, Lledó $\mathrm{S}$, Rodrigo JM. Risk factors for nonhepatic surgery in patients with cirrhosis. World J Surg. 2003;27(6):647-52.

10. Jakab F, Ráth Z, Sugár I, Ledniczky G, Faller J. Complications following major abdominal surgery in cirrhotic patients. Hepatogastroenterology. 1993;40(2):176-179.

11. Barmparas G, Cooper Z, Ley EJ, Askari R, Salim A. The effect of cirrhosis on the risk for failure of nonoperative management of blunt liver injuries. Surgery. 2015;158(6):1676-85.

12. Cook MR, Fair KA, Burg J, Cattin L, Gee A, Arbabi S, et al. Cirrhosis increases mortality and splenectomy rates following splenic injury. Am J Surg. 2015;209(5):841-7. doi: 10.1016/j.amjsurg.2015.01.009

13. Talving P, Lustenberger T, Okoye OT, Lam L, Smith JA, Inaba K, et al. The impact of liver cirrhosis on outcomes in trauma patients: a prospective study. J Trauma Acute Care Surg. 2013;75(4):699-703. doi: 10.1097/TA.0b013e31829a2c19

14. Gallstones and Laparoscopic Cholecystectomy. NIH Consens Statement. 1992;10(3):1-28.

15. McGillicuddy JW, Villar JJ, Rohan VS, Bazaz S, Taber DJ, Pilch NA, et al. Is cirrhosis a contraindication to laparoscopic cholecystectomy? Am Surg. 2015;81(1):52-55.

16. Shaikh AR, Muneer A. Laparoscopic cholecystectomy in cirrhotic patients. JSLS. 2009;13(4):592-596.

17. Hamad MA, Thabet M, Badawy A, Mourad F, Abdel-Salam M, AbdelRahman Mel-T, et al. Laparoscopic versus open cholecystectomy in patients with liver cirrhosis: a prospective, randomized study. J Laparoendosc Adv Surg Tech A. 2010;20(5):405-9. doi: 10.1089/ lap.2009.0476

18. Healthcare Cost and Utilization Project (HCUP) [database online]. Rockville, MD: Agency for Healthcare Research and Quality; 2011 Available from: https://www.hcup-us.ahrq.gov/nisoverview.jsp

19. Semenov YR, Starmer HM, Gourin CG. The effect of pneumonia on short-term outcomes and cost of care after head and neck cancer surgery. Laryngoscope. 2012;122(9):1994-2004.

20.Tsugawa K, Koyanagi N, Hashizume M, Tomikawa M, Ayukawa $\mathrm{K}$, Akahoshi K, et al. 0 A comparison of an open and laparoscopic appendectomy for patients with liver cirrhosis. Laparosc Endosc Percutan Tech. 2001;11(3):189-94.

21. Hamad MA, Thabet M, Badawy A, Mourad F, Abdel-Salam M, AbdelRahman Mel-T, et al. Laparoscopic versus open cholecystectomy in patients with liver cirrhosis: a prospective, randomized study. J Laparoendosc Adv Surg Tech A. 2010;20(5):405-9. doi: 10.1089/ lap.2009.0476

22. Poggio JL, Rowland CM, Gores GJ, Nagorney DM, Donohue JH. A comparison of laparoscopic and open cholecystectomy in patients with compensated cirrhosis and symptomatic gallstone disease. Surgery. 2000;127(4):405-11.

23. Rossi GL, Mentz R, Bertone S, Ojea Quintana G, Bilbao S, Im VM, et al. Laparoscopic peritoneal lavage for Hinchey III diverticulitis: is it as effective as it is applicable? Dis Colon Rectum. 2014;57(12):1384-90. doi: 10.1097/DCR.0000000000000252

24. Myers E, Hurley M, O'Sullivan GC, Kavanagh D, Wilson I, Winter DC. Laparoscopic peritoneal lavage for generalized peritonitis due to perforated diverticulitis. Br J Surg. 2008;95(1):97-101. 
25. Franklin ME Jr, Portillo G, Treviño JM, Gonzalez JJ, Glass JL. Long-term experience with the laparoscopic approach to perforated diverticulitis plus generalized peritonitis. World J Surg. 2008;32(7):1507-11. doi: 10.1007/s00268-007-9463-y

26. Li MZ, Lian L, Xiao LB, Wu WH, He YL, Song XM. Laparoscopic versus open adhesiolysis in patients with adhesive small bowel obstruction: a systematic review and meta-analysis. Am J Surg. 2012;204(5):77986.

27. Kelly KN, Iannuzzi JC, Rickles AS, Garimella V, Monson JR, Fleming FJ Laparotomy for small-bowel obstruction: first choice or last resort for adhesiolysis? A laparoscopic approach for small-bowel obstruction reduces 30-day complications. Surg Endosc. 2014;28(1):65-73. doi: 10.1007/s00464-013-3162-6

28. Chopra R, McVay C, Phillips E, Khalili TM. Laparoscopic lysis of adhesions. Am Surg. 2003;69(11):966-8.

29. Okamoto H, Wakana H, Kawashima K, Fukasawa T, Fujii H. Clinical outcomes of laparoscopic adhesiolysis for mechanical small bowel obstruction. Asian J Endosc Surg. 2012;5(2):53-8.

30. Siu WT, Leong HT, Law BK, Chau CH, Li AC, Fung KH, et al. Laparoscopic repair for perforated peptic ulcer: a randomized controlled trial. Ann Surg. 2002;235(3):313-319.

31. Bertleff MJ, Halm JA, Bemelman WA, van der Ham AC, van der Harst E, Oei HI, et al. Randomized clinical trial of laparoscopic versus open repair of the perforated peptic ulcer: the LAMA trial. World J Surg 2009;33(7):1368-1373.

32. Neeff HP, Streule GC, Drognitz O, Tittelbach-Helmrich D, Spangenberg HC, Hopt UT, et al. Early mortality and long-term survival after abdominal surgery in patients with liver cirrhosis. Surgery. 2014;155(4):623-32.

33. de Goede B, Klitsie PJ, Lange JF, Metselaar HJ, Kazemier G. () Morbidity and mortality related to non-hepatic surgery in patients with liver cirrhosis: a systematic review. Best Pract Res Clin Gastroenterol. 2012;26(1):47-59.

34. Choi SB, Hong KD, Lee JS, Han HJ, Kim WB, Song TJ, et al. Management of umbilical hernia complicated with liver cirrhosis: an advocate of early and elective herniorrhaphy. Dig Liver Dis. 2011;43(12):991-995.

35. Lehnert T, Herfarth C. Peptic ulcer surgery in patients with liver cirrhosis. Ann Surg. 1993;217(4):338-46

36. Friedman LS. (1999) The risk of surgery in patients with liver disease. Hepatology. 1999;29(6):1617-23.

37. Lin CS, Lin SY, Chang CC, Wang HH, Liao CC, Chen TL. Postoperative adverse outcomes after non-hepatic surgery in patients with liver cirrhosis. Br J Surg. 2013;100(13):1784-90.

38. Lopez-Delgado JC, Esteve F, Javierre C, Torrado H, Carrio ML, Rodríguez-Castro D, et al. Predictors of long-term mortality in patients with cirrhosis undergoing cardiac surgery. J Cardiovasc Surg (Torino). 2015;56(4):647-54

39. Garrison RN, Cryer HM, Howard DA, Polk HC Jr. Clarification of risk factors for abdominal operations in patients with hepatic cirrhosis. Ann Surg. 1984;199(6):648-655.

40. Rice HE, O'Keefe GE, Helton WS, Johansen K. Morbid prognostic features in patients with chronic liver failure undergoing nonhepatic surgery. Arch Surg. 1997;132(8):880-4. 